\title{
Current Status of Radiation Dose Levels in Conventional Pediatric Radiography: A Review Study
}

\author{
Abdelmoneim Sulieman \\ Radiology and Medical Imaging Department, College of Applied Medical Sciences, Prince Sattam bin Abdulaziz \\ University, Al-Kharj, Kingdom of Saudi Arabia \\ Email: abdelmoneim_a@yahoo.com
}

Received 26 August 2014; accepted 20 June 2015; published 23 June 2015

Copyright (C) 2015 by author and Scientific Research Publishing Inc.

This work is licensed under the Creative Commons Attribution International License (CC BY). http://creativecommons.org/licenses/by/4.0/

c) (7) Open Access

\begin{abstract}
Artificial sources of radiation account for approximately $14 \%$ of the annual radiation dose from all sources of radiation. Because of the increased lifetime risk per unit dose for children, radiographic procedures could lead to increase the radiogenic risk of cancer. This study intended to review the pediatric doses in planar radiography procedures and to assess different methods used to reduce the radiation dose for pediatric patients. Studies addressing pediatric dose optimization were identified from a search of the internet scientific databases. The search in literature was limited to journal articles that were written in English. The findings of the study illustrate that there are many available methods of dose reduction are available. The application of dose reduction methods will reduce the dose up to $75 \%$ of its current value. Training of staff is the cornerstone of patient dose optimization in pediatric radiology.
\end{abstract}

\section{Keywords}

Conventional Radiography, Pediatric Imaging, Radiation Risk, Medical Exposure, Effective Dose

\section{Introduction}

Planar radiograph (plain film or projection radiograph) is the practice of producing two-dimensional images using X-ray radiation. The radiographic images can be received on a film (screen film radiography (SFR)), special cassettes (computed radiography (CR)), or it may directly activate a matrix of solid-state detectors (digital radiography (DR)). SFR and CR systems utilize a cassette that houses the imaging receptor (film or imaging plate (IP)), while DR system captures the image directly onto a flat panel detector without the use of a cassette. Di- 
agnostic radiology is vital for the health care, but due to the radiation risks, radiation protection of the patient becomes an important issue. Medical exposure is the largest source of man-made exposure to ionizing radiation accounting for nearly $96 \%$ of all man-made radiation exposure to human and continues to grow substantially [1] [2]. Infants and children constitute $10 \%$ of the total number of radiological examinations [1] [3]-[6]. The number of pediatric diagnostic procedures conducted annually is currently above 300 million throughout the world [7]. Special attention should be given to infants and children because they are more sensitive to ionizing radiation than adults, having a risk of developing a radiation-induced cancer, hereditary effects or other serious disorders two to three times that of adults because of their greater cell proliferation rate and long life span expectancy, which means a greater potential for manifestation of possible harmful effects of radiation [1] [3]. The radiation risk is therefore highest in infancy and early childhood, in line with general growth patterns, and in adolescence, it gradually approaches the risk to which adults are exposed. Also, the use of X-ray equipment settings designed for adults can result in a larger radiation dose than necessary to produce a useful image for a smaller pediatric patient [8]. The International Commission on Radiation Protection (ICRP) [3] estimates that the risk coefficients for the average population are $5 \%$ and $1.3 \% \mathrm{~Sv}^{-1}$, whereas for children they are $13 \%$ and $4 \%$ $\mathrm{Sv}^{-1}$ for cancer effects and hereditary effects, respectively. Therefore, acceptable radiation protection measures are required in pediatric radiology. Even though the benefits derived from radiology procedures are beyond doubt, risks-benefits ratios need to be constantly monitored, as the use of ionizing radiation increases rapidly. Pediatric examinations require special attention in the justification process, optimization from unnecessary radiation exposure, since children are at greater risk of incurring stochastic effects. Pediatric doses should be kept as low as reasonably achievable, commensurate with the medical purpose. Pediatric radiology represents a special challenge for medical practitioners because the radiation doses vary widely among different institutions, and even for the same projection [9]-[12]. The dose variation may be due to patient characteristics (weight, body mass index (BMI), exposure factors (tube voltage $(\mathrm{kVp})$, tube current-time product (mAs), imaging technique, focus to film distance (FFD), film-screen speed, equipment type and processing performance). Additional factors such as poor beam collimation, inadequate devices for immobilization, lack of adequate quality control and the need for age-specific exposure factors based on appropriate anatomical parameters also affect patient doses. This study intended to review the pediatric doses in planar radiography procedures (chest, abdomen and spine, extremities and skull (dental and mammography are not applicable to pediatric populations, therefore they are not considered in this review)) and to assess different methods used to reduce the radiation dose for pediatric patients.

\section{Materials and Methods}

An inclusive literature review was carried out in order to quantify the radiation dose in different pediatric planar radiography procedures. Therefore, other imaging modalities such as computed tomography (CT) and interventional radiology procedures were excluded. The research strategy for this particular review used the database PubMed, Sage and ScienceDirect in additional to the available data on the internet from international organizations. The search in literature was limited to the journal articles that were written in English and published after 2004 to ensure that the literature being reviewed was recent and up to date. There were no restrictions on the country of origin where the publications were produced, which help to provide a range of opinions and experiences. Articles identified from the refined search results were further reviewed on an individual basis for content.

\section{Results and Discussion}

\subsection{Radiation Risk in Pediatric Radiology}

The biological effects of ionizing radiation (photons or charged particles) due to the absorption of energy at molecular level directly or indirect oxidative damage produced by water radiolysis. The biological effects are classified into two categories: Tissue reactions (deterministic or non stochastic effects) and cancer/hereditary effects (stochastic effects) [1] [3].

\subsection{Deterministic Effects}

Deterministic effects (or tissue reactions) of ionizing radiation are related directly to the absorbed radiation dose and the severity of the effect increases as the dose increases. A deterministic effect typically has a threshold (of the order of magnitude of 0.1 Gy or higher) under which the effect does not occur. Deterministic effects are 
based on tissue damage. However, deterministic effects of ionizing radiation do not need to be considered as a health hazard at the low doses delivered during diagnostic radiography procedures [1] [3].

\subsection{Stochastic Effects}

Stochastic effects occur without specific threshold of radiation doses because ionizing radiation is carcinogenic factor since it can act to initiate, promote and progress cellular changes that lead to cancer. The dose of radiation received by an individual affects the probability of cancer, but not its aggressiveness. The probabilistic nature of this risk means that children have more time to accumulate exposures and damage, and more time after exposure to develop the disease as illustrated in (Figure 1) [1] [3].

\subsection{Patient Dosimetry Methods}

Generally recommended measurable dose quantities are the entrance surface air kerma dose (ESAK) for individual radiographic projections and the kerma-area product (KAP) for complete $\mathrm{X}$ ray examinations. The ESAK can be directly measured with thermoluminescence dosimeters (TLDs), or it can be estimated on the basis of measured radiation output values for the X-ray tube. The total KAP from a complete examination, even when it involves both fluoroscopy and radiography, can be measured with a KAP meter and then compared directly against an appropriate reference level. Since the dose is critically dependent on patient size, it is recommended that measurements should be made for a representative sample of standard sized patients. The average dose of such a sample of each particular type of radiograph or examination would provide a good indication of the typical clinical practice in each room of an X ray department. The average doses should also be compared against national reference doses, in order to assess local performance [14].

The ESAK and the KAP are directly measurable quantities. They can be used for comparison against reference levels and for other quality assurance purposes, but they are not directly risk related quantities. Effective doses are needed in order to assess the population's collective effective dose arising from the medical use of radiation. Organ doses and/or the effective dose cannot be measured but can be estimated on the basis of measured ESAK or KAP values. Table 1 illustrates the radiation dose level from Planar imaging procedures.

The ICRP [15] has recommended the use of diagnostic reference level (DRL) in order to avoid radiation dose to the patient that does not contribute to the clinical purpose of a medical imaging task. This is accomplished by comparison between the numerical value of the diagnostic reference level (derived from relevant regional, national or local data) and the mean or other appropriate value observed in practice for a suitable reference group of patients or a suitable reference phantom. A reference group of patients is usually defined within a certain range of physical parameters (e.g. Height, weight). If an unselected sample of patients were used as a reference

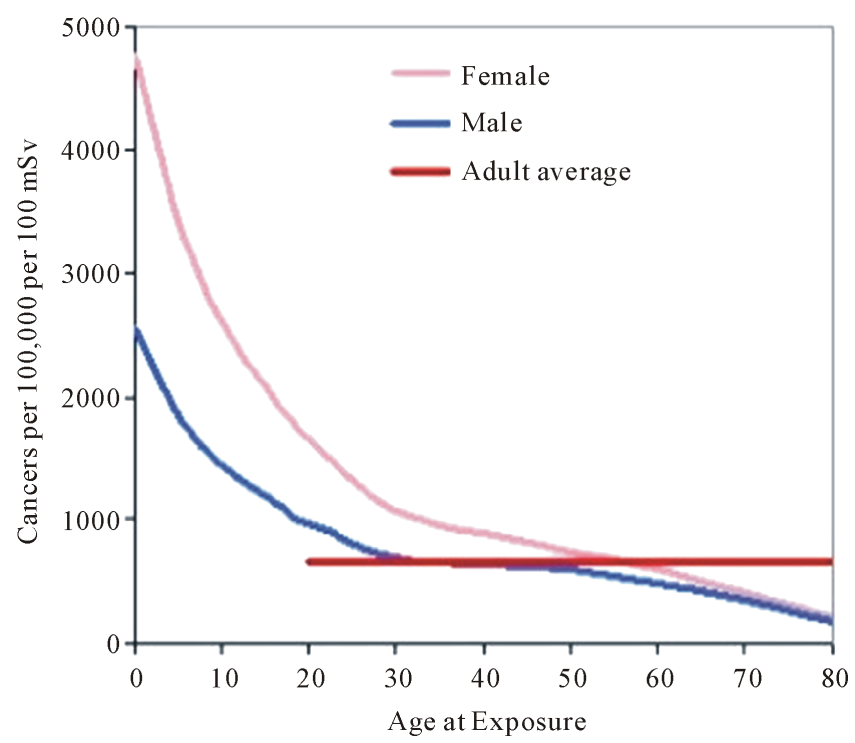

Figure 1. Lifetime incidence of cancer vs. age at exposure [13]. 
Table 1. Typical dose levels in pediatric radiology [16].

\begin{tabular}{cccccc}
\hline & \multicolumn{5}{c}{ ESAK $(\mu \mathrm{Gy})$} \\
\cline { 2 - 6 } Examination & 0 & 1 & Age & 10 & 15 \\
\cline { 2 - 6 } & 110 & 340 & 5 & 860 & 2010 \\
\hline Abdomen AP & 60 & 80 & 110 & 70 & 110 \\
Chest PA/AP & 170 & 350 & 510 & 650 & 1300 \\
Pelvis AP & $/$ & 600 & 1250 & $/$ & $/$ \\
Skull AP & $/$ & 340 & 580 & $/$ & , \\
Skull LAT & & &
\end{tabular}

*PA: Postero-anterior; AP: Antero-posterior.

group, it would be difficult to interpret whether the observed value of the sample is higher or lower than the diagnostic reference level. A diagnostic reference level does not apply to individual patients.

\subsection{Methods of Dose Reduction in Pediatric Imaging}

Lead apron will reduce the testicular absorbed dose by up to $95 \%$ [15]. Effective dose to ovaries protection is more difficult, but correct positioning of lead protection shields can result in a dose reduction to the ovaries of up to $50 \%$ [15]. Developing breast tissue is particularly sensitive to radiation. Therefore a PA chest technique is preferred as soon as the patient's ability to co-operate permits. Martin et al. [17] achieved a dose reduction for each age group, with $7 \%$ - 55\% decrease in critical organ dose without impairing image quality. Clinicians confirmed diagnostic image quality throughout the iterative process. Analysis of images acquired with preliminary and final exposure factors indicated an average visual grading analysis result of 0.5 , demonstrating equivalent image quality. The optimization process and final radiation doses are reported for Care stream computed radiography to aid other hospitals in minimizing radiation risks to children. A collaboration of multiple staff groups has resulted in significant reduction in the risk of radiation-induced cancer from radiographic X-ray exposure during childhood [1].

Pediatric procedures required lower exposure factors than adults to obtain an acceptable diagnostic radiograph due to their thinner body. Therefore, the X-ray machines should be capable to provide the necessary short exposure times with sufficient consistency, even with low target thicknesses [17]. X-ray examinations of adults must be performed with a tube filter of at least $2 \mathrm{~mm}$ aluminum. For children and adolescents, an additional tube filter of $1 \mathrm{~mm}$ aluminum $(\mathrm{Al})$ and 0.1 to $0.2 \mathrm{~mm}$ copper $(\mathrm{Cu})$ must be used. X-ray images must be as well collimated in order to reduce the scattered radiation. In addition to that, correct gonad protection must therefore be used according to the ICRP and food and drug administration guidelines [18] [19]. Organ shielding may be quite effective as it can lower the dose to the testes by about $95 \%$ and the dose to the ovaries by about $50 \%$ [17]. Organ shielding in radiology has become common practice and is recommended by national and international bodies [6] [18] [19]. The conditions for its application are: The gonads will lie in the primary X-ray field or within close proximity $(5 \mathrm{~cm})$, the clinical objectives of the examination will not be compromised and the patient has a reasonable reproductive potential. Table 2 reviews the available dose reduction techniques developed by different authors [2] [4] [6]-[19].

\subsection{Referral Criteria}

Referral criteria or justification of the procedures should base on clear clinical indications for pediatric patients. If the procedure is justified by a qualified medical practitioner, the techniques should be specifically tailored for pediatric patients, necessitating improvement in both equipment and staff training; and patient preparation guidelines, exposure factors, diagnostic guidance (reference) levels and image quality criteria should be developed. From a radiological protection perspective, clear justification of radiological examinations for children and young adults is essential. In addition, dose protocols and techniques have to be adapted to children and young adult patients while providing the required diagnostic information, thus optimizing protection. 
Table 2. Methods of pediatric patient protection in planar imaging.

\begin{tabular}{ccc}
\hline Parameters & Effects & Patient Dose \\
\hline High frequency generator & Improve the accuracy and & Reduce patient dose \\
Mobile X Ray & reproducibility of exposures & Increase patient dose \\
Protective shield & Long exposure time & Reduce patient dose \\
Additional filtration & Protection of gonads & Reduce patient dose \\
Automatic exposure control (AEC) & Absorption of soft radiation & Reduce patient dose \\
X-ray rooms & Manual selection of exposure factors & Avoid repetition \\
Fast screen-film combinations & Improve patient cooptation & Motion reduction \\
Digital radiography & Short exposure time & Avoid motion \\
Radiation field collimation & Short exposure time & Improve image quality \\
Reject analysis & Reduction of scatter radiation & Reduce the repetition rate \\
Patient immobilization & Image quality improvement & Reduce the repetition rate \\
Pulsed mode fluoroscopy & Image quality improvement & ALARA principles \\
\hline
\end{tabular}

\subsection{Diagnostic Reference Level}

A recommended dose for a radiologic study is usually expressed in DRL. This reference value is set to provide the lowest possible radiation dose that can produce images of sufficient diagnostic value. It is usually the third quartile value of the overall distribution of radiation doses to be applied in real practice [3] [6].

\subsection{Challenges in Pediatric Imaging}

Development of pediatric radiology comes with many challenges. Unlike adults, children cannot always understand a change of environment. Therefore, staff is usually required to wear colorful uniforms as opposed to a normal hospital uniform. It is also important to recognize that when a child is unwell, they follow their instincts, which is usually to cry and stay close to their parents. This presents a huge challenge for the radiography specialist, who must try to gain the child's trust and gain their cooperation. Once co-operation has been achieved there is another big challenge of keeping the child still for their imaging test. This can be very difficult for children in a lot of pain. Coercion and support from parents is usually enough to achieve this, however, in some extreme cases (such as MRI and CT), it may be necessary to sedate the child [20]-[23].

Another challenge faced is the radiation difference between an adult and child. Operators must use different equipment, setting for pediatric patients in order to reduce patient doses. Collaboration by several radiology, medical physics, pediatrics, and governmental organizations are essential to increase awareness of radiation safety issues in children and to provide education to all stakeholders caring for children on ways to decrease the ionizing radiation exposure in children [21]-[23]. There is information for parents that include basic information brochures that can be printed or downloaded that describe what is an x ray, what are the risks and benefits, and what can be done to decrease these risks [23].

\section{Conclusion}

Pediatric radiography has been steadily improved over the last years. Imaging and dose reduction are improved in order to assure that the patient will receive the lowest radiation dose without impairing image quality. Although there are many dose reduction measures helping in dose reduction in the literature, it is important to recognize that pediatric patients still receive a higher dose from unnecessary radiation. To successfully diagnose a pediatric condition, high quality images are needed to give a diagnosis. To achieve this requires creating an environment where a child is comfortable. This is one of the most essential elements to pediatric radiology. For im- 
aging departments which specialize in pediatric radiology, this is very easy as rooms can be tailored to suit a child's needs. There is no standard protocol for this procedure in all hospitals; thus, children receive a high dose of radiation in some causes.

\section{References}

[1] United Nations Scientific Committee on the Effects of Atomic Radiation (2008) Sources and Effects of Ionizing Radiation, UNSCEAR 2008 Report: Volume I: Sources-Report to the General Assembly Scientific Annexes A and B.

[2] Paterson, A., Frush, P.D. and Donnelly, L (2001) Helical CT of the Body: Are Settings Adjusted for Pediatric Patients? American Journal of Roentgenology, 176, 297-301. http://dx.doi.org/10.2214/ajr.176.2.1760297

[3] International Commission of Radiological Protection (1991) 1990 Recommendations of the International Commission of Radiological Protection. ICRP 60. Pergamon Press, Oxford.

[4] Sulieman, A., Theodorou, K., Vlychou, M., Kanavou, D., Fezoulidis, I. and Kappas, C. (2007) Radiation Dose Measurement and Risk Estimation for Paediatric Patients Undergoing Micturating Cystourethrography. The British Journal of Radiology, 80, 731-737. http://dx.doi.org/10.1259/bjr/16010686

[5] National Council on Radiation Protection and Measurements (1989) Exposure of U.S. Population from Diagnostic Medical Radiation. NCRP Report No. 100, Bethesda.

[6] European Commission (1996) Quality Criteria for Diagnostic Radiographic Images in Paediatrics. Report EUR 16261, Office for Official Publications of the European Communities, Luxembourg, 1-35.

[7] National Council on Radiation Protection and Measurements (2009) Ionizing Radiation Exposure of the Population of the United States. NCRP Report No. 160, Bethesda.

[8] Food and Drug Administration. Pediatric Dose Reduction Efforts. http://www.fda.gov/NewsEvents/Newsroom/PressAnnouncements/ucm303386.htm

[9] Sulieman, A., Vlychou, M., Tsougos, I. and Theodorou, K. (2011) Radiation Doses to Paediatric Patients and Comforters Undergoing Chest X-Rays. Radiation Protection Dosimetry, 147, 171-175. http://dx.doi.org/10.1093/rpd/ncr295

[10] Martin, L., Ruddlesden, R., Makepeace, C., Robinson, L., Mistry, T. and Starritt, H. (2013) Paediatric X-Ray Radia Tion Dose Reduction and Image Quality Analysis. Journal of Radiological Protection, 33, 621-633. http://dx.doi.org/10.1088/0952-4746/33/3/621

[11] Lateef, T.M., Grewal, M., McClintock, W., Chamberlain, J., Kaulas, H. and Nelson, K.B. (2009) Headache in Young Children in the Emergency Department: Use of Computed Tomography. Pediatrics, 124, e12-e17. http://dx.doi.org/10.1542/peds.2008-3150

[12] Alzen, G. and Benz-Bohm, G. (2011) Radiation Protection in Pediatric Radiology. Deutsches Ärzteblatt International, 108, 407-414.

[13] National Research Council Committee to Assess Health Risks from Exposure to Low Levels of Ionizing Radiation (2006) Health Risks from Exposure to Low Levels of Ionizing Radiation: BEIR VII Phase 2. National Academy of Sciences, National Academies Press, Washington DC.

[14] International Commission on Radiation Units and Measurements (ICRU) (2005) Patient Dosimetry for X Rays Used in Medical Imaging, ICRU Report 74, Volume 5, No. 2, Oxford University Press, Oxford.

[15] International Commission of Radiological Protection (2007) Recommendations of the International Commission of Radiological Protection. ICRP 103, Pergamon Press, Oxford.

[16] National Radiological Protecton Board (2002) Radiation Exposure of the UK Population from Medical and Dental X-Ray Examinations. NRPB-W4, Chilton.

[17] Sulieman, A., Barakat, H., Zailae, A., Abuderman, A. and Theodorou, K. (2015) Measurement of Patient Radiation Doses in Certain Urography Procedures. Radiation Protection Dosimetry, in Press. http://dx.doi.org/10.1093/rpd/ncv185

[18] Frantzen, M., Robben, M., Postma, A., Zoetelief, J., Wildberger, J. and Kemerink, G. (2012) Gonad Shielding in Paediatric Pelvic Radiography: Disadvantages Prevail over Benefit. Insights into Imaging, 3, 23-32. http://dx.doi.org/10.1007/s13244-011-0130-3

[19] International Commission on Radiological Protection (1982) Protection of the Patient in Diagnostic Radiology (ICRP Publication 34). Annals of the ICRP, 9, 18-23.

[20] Food and Drug Administration (FDA), 21 CFR 1000.50 (1976) Recommendation for the Use of Specific Area Gonad Shielding on Patients during Medical Diagnostic X-Ray Procedures. http://cfr.vlex.com/vid/1000-recommendation-gonad-shielding-patients-ray-19716085

[21] Lee, C.I., Haims, A.H., Monico, E.P., Brink, J.A. and Forman, H.P. (2004) Diagnostic CT Scans: Assessment of Pa- 
tient, Physician, and Radiologist Awareness of Radiation Dose and Possible Risks. Radiology, 231, 393-398. http://dx.doi.org/10.1148/radiol.2312030767

[22] Biological Effects of Ionizing Radiation (BEIR) VII (2006) Health Risks from Exposure to Low Levels of Ionizing Radiation Phase 1. National Academies Press, Washington DC.

[23] Brenner, D.J. and Hall, E.J. (2007) Computed Tomography-An Increasing Source of Radiation Exposure. New England Journal of Medicine, 357, 2277-2284. http://dx.doi.org/10.1056/NEJMra072149 